\title{
Erfahrungen des Rechtsvergleichs und verfassungsrechtliche Vorgaben in Deutschland
}

\author{
Peter A. Köhler
}

I. Zehn Jahre Rechtsvergleich: Die Arbeitsergebnisse der neuen „Fachgruppe“ der Gesellschaft für Rechtsvergleichung und der von der Bundesregierung eingesetzten „Studien-“ und „Fachkommission“"

1. Die Fachgruppe der Gesellschaft für Rechtsvergleich

2. Die Studienkommission für die Reform des öffentlichen Dienstrechts

3. Die Sachverständigenkommission Alterssicherung

II. Das Beamtentum in der Krise - krisenfest?

1. Der 39. Deutsche Juristentag 1951

2. Das Urteil des BVerfG zum Gesetz zu Art. 131 GG als Thema der Staatsrechtslehrertagung 1954 - Rückbesinnung auf die Staatsrechtslehrertagung 1931

III. Beamtentum, aktuelle Reformversuche und das Bundesverfassungsgericht

1. Beamtenrecht versus Beamtentum

2. „beachten“" versus „,berücksichtigen“ - „Modifizierung“ versus „Veränderung“

3. „Traditionsbildender Zeitraum“ versus Regelungsbedürfnisse der Gegenwart 
Alle traditionellen Alterssicherungssysteme der sozialen Sicherheit sind mehr oder weniger vom demografischen Wandel der Bevölkerung betroffen oder werden es in absehbarer Zeit sein. Dieses Problem prägt seit Jahren die internationale sozialpolitische Diskussion. Weitaus die meisten der dazu erarbeiteten Studien galten und gelten nicht allein aus pragmatischen Gründen, sondern wegen ihrer objektiv großen gesellschaftlichen Bedeutung den jeweiligen allgemeinen Sicherungssystemen. ${ }^{1}$ Doch betreffen die synchron mit der längeren Lebenserwartung wachsenden Laufzeiten der Leistungen der Alterssicherung dort, wo es diese gibt, natürlich auch Sondersysteme für bestimmte Bevölkerungsgruppen, etwa berufsständische Systeme (z.B. für die freien Berufe) oder die Beamtenversorgung in Deutschland. ${ }^{2}$

Diese sind im Verhältnis zu den allgemeinen Systemen längst nicht mehr marginal. So gab es in Deutschland in der Beamtenversorgung im Jahr 1980 insgesamt (also einschließlich der Witwen- und Waisenpensionen und der Versorgungsempfänger nach dem „131-Gesetz“) 1,09 Mio. Versorgungsempfänger. ${ }^{3}$ Bis 2008 stieg diese Zahl auf 1,47 Mio., das waren 1,3\% mehr als im Vorjahr und $20 \%$ mehr als noch $1990 .{ }^{4}$ Alle Prognosen weisen auf ein ähnliches Ansteigen in den kommenden Jahren hin. ${ }^{5}$ Für die Staaten der EU gilt bei allen sonstigen Unterschieden, daß das Altersprofil der Bediensteten zu einer „Alterung des öffentlichen Sektors“6 geführt hat und daß deshalb die Zahl der Versorgungsempfänger in gleicher Weise zunehmen wird.

1 Die dazu vorliegenden nationalen und internationalen Studien unterschiedlicher wissenschaftlicher Disziplinen sind längst nicht mehr überschaubar; hier sei exemplarisch auf die rechtsvergleichenden Studien von Zacher (Hrsg.), Alterssicherung im Rechtsvergleich, 1991, Bd. 11 der Studien aus dem Max-Planck-Institut für ausländisches und internationales Sozialrecht, und von Reinhard (Hrsg.), Demographischer Wandel und Alterssicherung, Rentenpolitik in neun europäischen Ländern und den USA im Vergleich, 2001, Bd. 22 der Studien aus dem Max-Planck-Institut für ausländisches und internationales Sozialrecht, verwiesen, in denen mit Deutschland, Frankreich, Schweden, der Schweiz und dem Vereinigten Königreich auch ein Teil der vorliegend untersuchten Länder erfaßt wurden.

2 Siehe mit umfänglichem Zahlenmaterial Färber/Funke/Walther, Die Entwicklung der Beamtenversorgung in Deutschland seit 1992 und künftige Finanzierungsprobleme der Gebietskörperschaften, DÖV 2009, S. 133 ff. Zur aktuellen Diskussion des Einbezugs der Selbständigen in die gesetzliche Rentenversicherung s. die Beiträge in Sozialer Fortschritt 2009, Heft 9-10, insbes. die rechtsvergleichende Übersicht von Wirth/Müllenmeister-Faust, Die Alterssicherung Selbständiger in Deutschland und in Europa - Stand und Perspektiven, S. 210 ff.

3 Zacher, Versorgung der Beamten, Richter und Soldaten, in: Sachverständigenkommission Alterssicherungssysteme, Berichtsband 2, Darstellung der Alterssicherungssystem und die Besteuerung von Alterseinkommen, 1983, S. 127, 140.

4 Schwahn, Entwicklungen im öffentlich-rechtlichen Alterssicherungssystem, in: Statistisches Bundesamt (Hrsg.), Wirtschaft und Statistik 1/2009, S. 85.

5 Ders., ebda., S. 86.

Der Wegfall von Beamtenplanstellen durch Privatisierung (Bahn, Post, kommunale Dienste) läßt zwar eine gewisse Entspannung erwarten, freilich nicht in der unmittelbaren Zukunft.

6 Demmke, Die europäischen öffentlichen Dienste zwischen Tradition und Reform, 2005, S. 32, 37. Diese Prognose gilt auch für Länder mit ,einer relativ hohen Geburtenrate“. Aktuell wird etwa für Schweden festgestellt, daß binnen sechs Jahren jeder vierte Angestellte im öffentlichen Dienst das 
Angesichts der kontinuierlich wachsenden Bedeutung des Gemeinschaftsrechts stellt sich abgesehen davon die Frage, ob für die nationalen Strukturen des öffentlichen Dienstrechts eine „Angleichungstendenz“ festgestellt werden kann. ${ }^{7}$ Schließlich stehen die Institutionen der EU selbst, ebenso wie auch und gerade viele der traditionsreichen Internationalen Organisationen vor der Aufgabe, die Verpflichtungen aus den Versorgungssystemen der internationalen Beamten so zu gestalten, daß deren finanzielle Belastung nicht langfristig die Bereitschaft der Mitgliedstaaten übersteigt, dafür die Beiträge zu tragen.

\section{Zehn Jahre Rechtsvergleich: Die Arbeitsergebnisse der neuen „Fach- gruppe“ der Gesellschaft für Rechtsvergleichung und der von der Bun- desregierung eingesetzten ,Studien-“ und „Fachkommission“}

Die Erwartung, eine für das Recht des öffentlichen Dienstes notwendige „Ausrichtung auf die Zukunft" 8 durch Rechtsvergleichung fördern zu können, ist nicht erst die Folge des aktuell wachsenden Problemdrucks - sie war (zumindest theoretisch) bereits seit den 1970er Jahren evident.

\section{Die Fachgruppe der Gesellschaft für Rechtsvergleich}

Ein Beleg dafür ist das Aufgreifen des Themas durch die Wissenschaft. Nicht zuletzt wegen des Phänomens des seit den 1960er Jahren europaweit zu konstatierenden kontinuierlichen Anwachsens des aktiven öffentlichen Dienstes, verbunden mit dem zwar zeitverschoben, aber mit Sicherheit parallel dazu einsetzenden Wachsen der Versorgungslasten, beschloß die Gesellschaft für Rechtsvergleichung, als sie 1979 eine eigene Fachgruppe für Arbeits- und Sozialrecht gründete, „Soziale Sicherheit im öffentlichen Dienst" als deren erstes Arbeitsthema. ${ }^{9}$

Rentenalter erreicht haben wird, vgl. Orrenius, Var fjärtde statligt anställd är pensionerad om sex år, Riksdag \& Departement 30/2009, S. 12.

7 Demmke, Reformen der Personalpolitiken in den öffentlichen Diensten der EU-Mitgliedstatten - Europäisierung oder Differenzierung?, in: Magiera/Sommermann/Ziller (Hrsg.), Verwaltungswissenschaft und Verwaltungspraxis in nationaler und transnationaler Perspektive, FS Siedentopf, 2008, S. $551 \mathrm{ff}$.

8 Mayer, Vorwort zu Bd. 1. der Berichte der Studienkommission für die Reform des öffentlichen Dienstrechts, 1973, S. 17.

9 Zacher/Bullinger/Igl (Hrsg.), Soziale Sicherheit im öffentlichen Dienst in der Bundesrepublik Deutschland, den Niederlanden, Schweden, Belgien und den Europäischen Gemeinschaften, 1982. 
In der Dokumentation der Tagung wurde einleitend die besondere Problemkombination benannt, die bei diesem Vergleichsgegenstand zu bewältigen ist. Damals galt wie heute:

„Die soziale Sicherheit im öffentlichen Dienst ist ein Problembereich, in dem sachlichfunktionale Gesichtspunkte und historische Entwicklungen zu eigentümlichen Befunden führen.“

Daraus wurde die Fragestellung entwickelt,

„ob nicht aus der Besonderheit des Dienstherrn und der Aufgaben eine Besonderheit des Dienstrechts folgt, und ob nicht aus dieser Besonderheit des Dienstrechts ... eine Besonderheit der sozialen Sicherung folgen und also bleiben muß." 10

Die neue Fachgruppe erarbeitete nicht nur eigene Landesberichte, sondern bezog das Dienstrecht der europäischen Beamten bereits mit ein. Der daraufhin dieses internationale Recht und die diversen nationalen Rechte vergleichende Generalbericht verstand sich weniger als Darstellung definitiver Ergebnisse, sondern als eine betont zurückhaltende Skizze der Unterschiede. ${ }^{11}$

Dabei konnte die Fachgruppe bei ihrer Arbeit auf die Ergebnisse einer schon 1970 eingesetzten „Studienkommission für die Reform des öffentlichen Dienstrechts“12 zurückgreifen und zudem in Kenntnis dessen arbeiten, daß die Bundesregierung im Sommer 1981 erneut eine „Sachverständigenkommission Alterssicherung"13 einberufen hatte - das Thema fand also weites Interesse und war keineswegs erschöpft.

\section{Die Studienkommission für die Reform des öffentlichen Dienstrechts}

Die 1973 vorgelegten Berichte der Studienkommission enthielten eine Reihe von Reformvorschlägen (u.a. Statusregelung, Berufszugang und Berufsweg, Bezahlung/Besoldung, Fürsorgeleistung und Versorgung), deren ganz überwiegende Mehrzahl freilich politisch nicht realisiert wurde. Offenbar griffen viele der Vorschläge zu weit: So wäre die in der mit führenden Experten besetzten Kommission mehrheitlich getroffene Empfehlung, alle Beschäftigungsverhältnisse des öffentlichen Dienstes gesetzlich zu regeln, auf eine Verbeamtung des gesamten Sektors hinausgelaufen. Doch bestand nicht nur in der Kommission weitgehende Einigkeit darüber, daß die dienstrechtliche Dreiteilung in Arbeiter, Angestellte und Beamte von der Entwicklung überholt sei, und daß das

10 Dies., ebda., S. 5: Zum Gegenstand dieses Bandes.

11 Tomandl, Generalbericht, in: Zacher/Bullinger/Igl (Hrsg.), Soziale Sicherheit im öffentlichen Dienst (Fußn. 9), S. 391 ff.

12 Die Ergebnisse wurden von der Studienkommission für die Reform des öffentlichen Dienstrechts durch verschiedene Herausgeber veröffentlicht: Berichte der Studienkommission für die Reform des öffentlichen Dienstrechts, 12 Bde., 1973.

13 Sachverständigenkommission Alterssicherungssysteme, Gutachten mit zwei Anlagebänden, veröffentlicht durch die Bundesregierung und den Bundesminister für Arbeit und Sozialordnung, 1983. 
Dienstrecht sich stärker als bisher an der Funktion und an der Leistung des jeweiligen öffentlich Bediensteten zu orientieren habe.

Die Frage der verfassungsrechtlichen Grenzen einer Reform des öffentlichen Dienstrechts wurde in mehreren eigenständigen Gutachten diskutiert.

Forsthoff hob u.a. hervor, bei der ,gebotenen konservierenden Betrachtung“ sei die „Entscheidung für den lebenslänglich angestellten Berufsbeamten (...) die evidente Folge aus dieser Sicht.“14 v. Münch führte nicht weniger als elf Grundsätze für die „ohne Verfassungsänderung stets zu berücksichtigenden (...) hergebrachten Grundsätze des Berufsbeamtentums" auf, 15 Schick hielt eine ersatzlose Streichung der Abs. 4 und 5 des Art. 33 GG für „unzulässig, weil die Beamtenverhältnisse nicht in einen rechtsleeren Raum entlassen werden können,"16 - eine Ansicht, die Ule noch entschiedener formulierte:

„Art 33 Abs. 4 und 5 GG können weder aufgehoben werden noch in wesentlichen Punkten geändert werden." 17

Thieme setzte sich gründlich mit dem Begriffsinhalt der ,,hergebrachten Grundsätze des Berufsbeamtentums" auseinander. Er hob die angesichts der jüngsten deutschen Geschichte besondere Problematik der Bestimmung dessen hervor, was denn als „hergebracht" anerkannt und deshalb als bewahrenswert gelten kann. ${ }^{18}$ Weiter stellte er heraus:

„Über die Bedeutung des Begriffes ,Grundsätze’ besteht weder in der Rechtsprechung noch in der Literatur Klarheit.“

Er kritisierte das Bundesverfassungsgericht (BVerfG), weil dem die Begriffe „Struktur“, „Prinzip“ und „Kernbestand“ nicht genügten, weshalb das Gericht diese „,noch zu einem ,Kernbestand an Strukturprinzipien' verbindet." 19 Dies erkläre nichts und ebenso unklar bleibe schließlich, ,was unter, Wesen des Beamtentums’ zu verstehen ist.“20

14 Forsthoff, Rechtsgutachten, in: Forsthoff/v. Münch/Schick/Thieme/Ule/Mayer (Hrsg.), Verfassungsrechtliche Grenzen einer Reform des öffentlichen Dienstrechts, Studienkommission für die Reform des öffentlichen Dienstrechts, 1973, Bd. 5, S. 69.

15 v. Münch, in: Forsthoff/v. Münch/Schick/Thieme/Ule/Mayer (Hrsg.), Verfassungsrechtliche Grenzen einer Reform des öffentlichen Dienstrechts (Fußn. 14), S. 163.

16 Schick, in: Forsthoff/v. Münch/Schick/Thieme/Ule/Mayer (Hrsg.), Verfassungsrechtliche Grenzen einer Reform des öffentlichen Dienstrechts (Fußn. 14), S. 293.

17 Ule, in: Forsthoff/v. Münch/Schick/Thieme/Ule/Mayer (Hrsg.), Verfassungsrechtliche Grenzen einer Reform des öffentlichen Dienstrechts (Fußn. 14), S. 555.

18 Thieme, in: Forsthoff/v. Münch/Schick/Thieme/Ule/Mayer (Hrsg.), Verfassungsrechtliche Grenzen einer Reform des öffentlichen Dienstrechts (Fußn. 14), S. 317.

19 Ders., ebda., S. 321. Vielleicht liegt auch da die Problematik darin, daß die Diskussion in verschiedenen Sprachen geführt wird. Siehe etwa die durchweg von Betriebswirtschaftlern, Bankkaufleuten und Wirtschaftsingenieuren geschriebenen Beiträge in Suhlrie (Hrsg.), Öffentlich-private Partnerschaften, 2009. Darin etwa Rabitzsch/Rüschen, Anreizmechanismen in der öffentlichen Verwaltung, S. 131 ff., die „für die Mitarbeiter des öffentlichen Sektors“ (offen bleibt, ob damit Beamte gemeint sind) Instrumente des „New Public Management“ wie Wettbewerb, Leistungsvergleiche, Benchmarking (Partner, Projekte, Parameter), ergänzt durch eine moderne, - auch Fehler verzeihende - 
Im gemeinsam erarbeiteten Ergebnis, in dem sie die Einzelfragen des Gutachtenauftrags abhandelten, kamen die Gutachter u.a. zur Auflistung von immerhin 18 „hergebrachten Grundsätze des Berufsbeamtentums“, die ,im Grundsatzkern (!) unverzichtbar sind“, darunter (Nr. 16) die ,amtmäßige Versorgung des Beamten und seiner hinterbliebenen Familienmitglieder auf der Grundlage der zuletzt (!) innegehabten Amtsbezüge“ 21 und zu der juristisch klugen, sonst aber wenig hilfreichen Feststellung:

„Die Frage der Zulässigkeit einer Änderung der Abs. 4 und 5 des Art. 33 GG könnte nur

behandelt werden, wenn ein bestimmter Änderungsvorschlag bereits vorliegen würde." 22

Auch wenn man mehrheitlich dann doch einen Rechtsgrund nicht erkennen wollte, „,wonach Art. 33 Abs. 4 und 5 GG nicht ersatzlos aufgehoben werden könnten“, so ergäben sich für das öffentliche Dienstrecht ,insbesondere aus Art. 20 und 1 GG weitere schwer überwindbare Schranken." 23

Diesen Befund einer „grundrechtsgleichen Position“ reflektiert als Ergebnis seines Rechtsvergleichs auch der Generalberichterstatter der neuen Fachgruppe der GfR, in dem zusammenfassend feststellt wird, daß unter den Vergleichsländern nur die Bundesrepublik Deutschland eine Verfassungsgarantie des Berufsbeamtentums kennt, „die jeder grundlegenden Umgestaltungsabsicht deutliche Schranken setzt.“"24

\section{Die Sachverständigenkommission Alterssicherung}

Die ein Jahr später veröffentlichten Gutachten der „Sachverständigenkommission Alterssicherung" sind ebenfalls das Ergebnis einer rechtsvergleichenden Untersuchung nach dem Gutachtensauftrag freilich national auf die in der Bundesrepublik etablierten Systeme beschränkt, also auf ,einen systematischen Vergleich aller staatlich finanzierten und geförderten Alterssicherungssysteme" und mit dem hochgesteckten Ziel,

Managementkultur" empfehlen (S. 145). Ganz ähnlich die Texte von Pfnür/Schetter/Schöbener, Risikomanagement bei Public Private Partnerships, 2009.

20 Thieme, in: Forsthoff/v. Münch/Schick/Thieme/Ule/Mayer (Hrsg.), Verfassungsrechtliche Grenzen einer Reform des öffentlichen Dienstrechts (Fußn. 14), S. 317.

21 Forsthoff/v. Münch/Schick/Thieme/Ule/Mayer (Hrsg.), Verfassungsrechtliche Grenzen einer Reform des öffentlichen Dienstrechts (Fußn. 14), Teil D Ergebnis, S. 699-700. Die Aufzählung reicht vom Definitionsversuch des Beamtenverhältnisses ,als ein öffentlichrechtliches Dienstverhältnis in Form eines besonderen Gewaltverhältnisses mit Funktionsvorbehalt“ (Nr. 1), über das „Laufbahnprinzip“ (Nr. 6), das „Lebenszeitprinzip und Hauptberuflichkeit sowie fachliche Vorbildung“ (Nr. 7), über besonders ausgestaltete Amtshaftung (Nr. 12), „Streikverbot“ (Nr. 14), bis zum „Recht auf Beamtenvertretungen, Vereinigungsfreiheit und Freiheit der politischen Gesinnung“ (Nr. 18).

22 Dies., ebda., S. 699.

23 Dies., ebda., S. 699.

24 Tomandl, in: Zacher/Bullinger/Igl (Hrsg.), Soziale Sicherheit im öffentlichen Dienst (Fußn. 9), S. 394. 
„Vorschläge für eine etwaige Neuregelung zu erarbeiten, die insgesamt nicht mit Mehraufwendungen verbunden sein sollen“. 25

Die vergleichende Analyse auf der Grundlage kompetenter Darstellungen der diversen, in der Bundesrepublik etablierten Systeme ist bis heute über weite Strecken keineswegs überholt. ${ }^{26}$ Das Gutachten Rulands betreffend die „Möglichkeiten und Grenzen einer Annäherung der Beamtenversorgung an die gesetzliche Rentenversicherung"27 enthält eine beeindruckende Bestandsaufnahme des damals (und hinsichtlich der Beamtenversorgung weithin auch aktuell noch) geltenden Rechts. Was 1983 wie schon leise, aber doch noch nicht vollständig hoffnungslose Resignation klang:

„Ein sinkendes Rentenniveau läßt aber das ,Traumziel' der Rentenversicherung, das Niveau der Beamtenversorgung einzuholen, in unerreichbare Ferne rücken." 28

führte zu der ,,von einer großen Mehrheit der Kommission“ getragenen Empfehlung:

„künftig auch die Beamten - wie die Versicherten der Rentenversicherung - schrittweise an der Finanzierung ihrer Alterssicherung zu beteiligen und entsprechende Gehaltsanteile der Beamten in ihren Bezügen offen auszuweisen. Dadurch soll ein tragender Gedanke der Alterssicherung verwirklicht werden: nämlich die Beteiligung aller Erwerbstätigen an der Finanzierung ihrer Alterssicherung innerhalb des jeweiligen Systems und die ausgewogene Beteiligung aller Gesicherten insbesondere an den aus der demographischen Entwicklung resultierenden Belastungen." 29

Der Gesetzgeber kam dieser Empfehlung ebenso wenig nach, wie er zehn Jahre zuvor die Vorschläge der „Studienkommission“ aufgegriffen hatte oder wie er - auf irgendeine nachweisbare Weise - die Arbeit der Fachgruppe der GfR zur Kenntnis genommen hat. Die (heimliche) Hoffnung, durch einen Rechtsvergleich dem Gesetzgeber nützlich werden zu können, ${ }^{30}$ wurde einmal mehr enttäuscht.

25 Der Beschluß der Bundesregierung, eine Sachverständigenkommission mit dem zitierten Auftrag einzusetzen, ist abgedruckt in: Sachverständigenkommission Alterssicherungssysteme, Berichtsband 1, Vergleich der Alterssicherungssysteme und Empfehlungen der Kommission, 1983, S. 17 ff.

26 Sachverständigenkommission Alterssicherungssysteme, Berichtsband 2, insbes. die Beiträge von Kolb, Gesetzliche Rentenversicherung, S. 7 ff., und Zacher, Versorgung der Beamten, Richter und Soldaten, S. 127 ff., die beide auch eine ebenso knappe wie präzise Darstellung der historischen Entwicklung beinhalten.

27 Ebda., Anlageband B.

28 Ruland, Sachverständigenkommission Alterssicherungssysteme (Fußn. 25), S. 19 Rdnr. 10.

29 Ders., ebda., Vergleich der Alterssicherungssysteme und Empfehlungen der Kommission, S. 144. Die Reformvorschläge sind deshalb über die Jahre bescheidener geworden. Ruland, Die Beamtenversorgung, in: Ruland/Rürup (Hrsg.), Alterssicherung und Besteuerung, 2008, S. 96, prognostiziert z.B. 2008, daß die bislang beitragsfreie Versorgung bei der Bestimmung des Gehaltsniveaus berücksichtigt werden müsse.

30 Zacher, Vorfragen zu den Methoden der Sozialrechtsvergleichung, in: v. Maydell/Eichenhofer (Hrsg.), Abhandlungen zum Sozialrecht, 1993, S. 329-375 (330), reflektiert die mögliche politischgesetzgeberische Nützlichkeit rechtsvergleichender Arbeit realistisch, also zurückhaltend - wenn man denn das Ziel „Erfassen, Verstehen und Bewerten von Recht“ nicht vorab als hinreichenden rechtstheoretischen Nutzen anerkennen will. 


\section{Das Beamtentum in der Krise - krisenfest?}

\section{Der 39. Deutsche Juristentag 1951}

Dabei ist es nicht so, daß für das deutsche Beamtentum über die Jahre kein Diskussions- und Handlungsbedarf bestanden hätte. Bei den Verhandlungen des 39. Deutschen Juristentags 1951 diskutierte die öffentlich-rechtliche Abteilung die Frage:

„Inwieweit läßt Art. 33 Abs. 5 des Grundgesetzes eine Reform des Beamtenrechts zu?“31

Noch vor den Referaten gab der Präsident des Juristentags der Diskussion die Richtung vor. Er wies auf die „Nuance“ hin, daß im Grundgesetz nicht vom „Berufsbeamtenrecht“, sondern vom „Berufsbeamtentum“ die Rede sei. Letzteres sei mit Art. 33 Abs. 5 GG gemeint, was „eine Parallele in Art 19 Abs. 2 GG findet", wonach Grundrechte wohl eingeschränkt, nicht aber in ihrem Wesensgehalt angetastet werden dürfen. In gleicher Weise seien

„die hergebrachten Grundsätzen des Berufsbeamtentums zu verstehen. Also: Reform gewiß; aber immer muß der Wesensgehalt des Berufsbeamtentums unangetastet bleiben." 32

Die Referate von Grewe und Fischbach standen beide erkennbar unter dem Eindruck des Wunsches nach „Neuanfang nach dunkler Zeit“, was freilich auch hinsichtlich des Berufsbeamtentums primär auf die Rückbesinnung auf die Rechtslage vor 1933 hinauslief, 33 allerdings mit dem Unterschied, daß von der überkommenen „Repräsentationsfunktion des Beamten“ Abstand genommen wurde. ${ }^{34}$ Hervorgehoben wurde, daß das Berufsbeamtentum nach dem „Bonner Grundgesetz“, anders als nach der ,institutionellen Garantie“ der Weimarer Reichsverfassung, ,die Eigenschaft einer wesentlichen Verfassungseinrichtung erhalten hat.“35 Man begrüßte dies, weil nur eine „starke rechtliche

31 Ständige Deputation des Deutschen Juristentags (Hrsg.), Verhandlungen des Neununddreissigsten (außerordentlichen) Deutschen Juristentags in Stuttgart 1951, 1952, Kap. D 2; s. auch die ausführlichen Berichte in der JZ 1951, S. 663 ff., und der NJW, 1951, S. 753.

32 Loening, in: Ständige Deputation des Deutschen Juristentags (Hrsg.), Verhandlungen DJT (Fußn. 31), S. D 2.

33 Dies trifft nicht nur für die Juristen dieser Zeit zu, auch für die politischen Parteien, Gewerkschaften, Verbände etc. bestand in der Nachkriegszeit der „Neuanfang“ weitgehend im Rückgriff auf die Zeit vor Hitler, s. Köhler/v. Maydell, 1945-1949, Die Zeit der Besatzungszonen, Sozialpolitik zwischen Kriegsende und der Gründung zweier deutscher Saaten, in: BM für Arbeit und Soziales/Bundesarchiv (Hrsg.), Geschichte der Sozialpolitik in Deutschland seit 1945, Band 2, 2006, S. $945 \mathrm{ff}$.

34 Grewe, in: Ständige Deputation des Deutschen Juristentags (Hrsg.), Verhandlungen DJT (Fußn 31), S. D 29, Thesen, Nr. I c).

35 Fischbach, in: Ständige Deputation des Deutschen Juristentags (Hrsg.), Verhandlungen DJT (Fußn. 31), S. D 34. Fischbach wird als Autor der führenden Kommentare zum Beamtenrechtsänderungsgesetz vom 30. Juni 1933 und des Deutschen Beamtengesetzes 1937 vom BVerfG in BVerfGE 
Fundierung“ gewährleisten könne, daß das Berufsbeamtentum „künftig parteipolitischen und gewerkschaftlichen Angriffen gegenüber standhalten kann." 36 Die von Grewe vorgetragene Hauptthese fand nicht nur über den Juristentag hinaus Beifall, ${ }^{37}$ sie deutet auch einen vorsichtigen Wandel im Denken an: Zweck der institutionellen Garantie des Berufbeamtentums sei nicht,

„sämtliche hergebrachten Grundsätze des Beamtenrechts zu konservieren, sondern vielmehr eine rechtlich, geistig und soziologisch fundierte Institution unseres öffentlichen Lebens in ihrer Ganzheit zum gesetzlichen Leitbild zu erheben."38

Ähnlich beurteilten die Zeitgenossen den 1951 von der Bundesregierung dem Bundesrat vorgelegten Entwurf des Bundesbeamtengesetzes, der im Wesentlichen am bisherigen Beamtenrecht festhielt. Immerhin:

„Neuerungen in der Richtung auf eine fortschrittliche Auflockerung werden in vorsichtigster Form eingeführt. “39

Man konnte dies auch als eine indirekte Bestätigung der „Identitätstheorie“ 40 verstehen, nach der der Deutsche Staat (und mit ihm seine Beamtenschaft) zumindest als Idee über die Kapitulation vom 8. Mai 1945 weiterbestand.

3, 58, 90 ausführlich zum Beleg der ideologischen Gleichschaltung des Berufsbeamtentums in der NS-Zeit zitiert.

36 Ders., ebda., S. D 41. Weiter führt er in offenbar zeitloser Gültigkeit aus (S. D 47): „Die Frau konnte sagen: 'Mein Mann ist Beamter!' - und, in zweiter Linie natürlich, der wichtige Versorgungsgedanke. (...) Dieser Punkt ist für das künftige Beamtenrecht, wenn es wirklich reformiert werden soll, von ganz wesentlicher Bedeutung."

Kenntner, Sinn und Zweck der Garantie des hergebrachten Berufsbeamtentums, DVB1 2007, S. 1321, 1326, plädiert noch heute für die ,parteipolitische Neutralisierung“ des Berufsbeamtentums und zitiert dafür aus den Materialien des Parlamentarischen Rats den Abgeordneten Dr. Strauß (CSU), dem damals die Verankerung des Prinzips der Gesetzmäßigkeit der Verwaltung ,ausgezeichnet gefallen“ hat und der betonte, daß „Gesetzmäßigkeit und parteipolitische Neutralität der Verwaltung (...]) am besten durch Berufsbeamte gesichert werden.“

37 Der ganze Text wurde im Bericht der JZ 1951 (Fußn. 31) abgedruckt.

38 Grewe, in: Ständige Deputation des Deutschen Juristentags (Hrsg.), Verhandlungen DJT (Fußn. 31), S. D 29, Thesen, Nr. I a).

39 Wessel, Zum Entwurf des Bundesbeamtengesetzes, JZ 1951, S. 571-572. Aus historischer Perspektive Benz, Versuche zur Reform des öffentlichen Dienstes in Deutschland 1945-1952, Vierteljahreshefte für Zeitgeschichte 2/1981, S. 216 ff.

$40 \mathrm{Zu}$ dieser s. deren (Mit-)Initiatoren Grewe, Ein Besatzungsstatus für Deutschland, 1948, und Kaufmann, Deutschlands Rechtslage unter der Besatzung, 1948. Scheuner, Die staatsrechtliche Kontinuität in Deutschland, DÖV 1950, S. 481 ff., sprach davon, „daß das Reich als Dienstherr der Reichsbeamten erhalten geblieben ist.“ (S. 484). Zur Gegenmeinung der vollständigen organisatorischen Diskontinuität s. Kelsen, The International Legal Status of Germany to be established immidiately upon Termination of the War, American Journal of Law, Vol. 38/1945, S. 689 ff., und ders., The Legal Status of Germany According to the Declaration of Berlin, ebda., S. 518 ff. Mit historischem Abstand Distelkamp, Rechts- und verfassungsgeschichtliche Probleme zur Frühgeschichte der Bundesrepublik, JuS 1980, S. 401 ff., 480 ff. und 790 ff.; Stolleis, Rechtsordnung und Justizpolitik 1945-1949, in: Horn, (Hrsg.), Europäisches Rechtsdenken in Geschichte und Gegenwart, Festschrift für Coing. Bd. 1, 1982, S. 383 ff.; eine Übersicht über die politischen Hintergründe des Diskurses gibt Perels, Die Restauration der Rechtslehre nach 1945, Kritische Justiz 1984, S. 359 ff. Rechtshis- 


\section{Das Urteil des BVerfG zum Gesetz zu Art. 131 GG als Thema der Staatsrechtsleh-} rertagung 1954 - Rückbesinnung auf die Staatsrechtslehrertagung 1931

Diese „fortschrittlichen Auflockerungen“ waren also nicht der eigentliche Grund, weshalb dann auf der Tagung der deutschen Staatsrechtslehrer 1954 neben dem Bericht „Der deutsche Staat im Jahr 1945 und seither“ das zu dieser Zeit naheliegende Thema „Das Berufsbeamtentum und die Staatskrisen“ zur Verhandlung stand. ${ }^{41}$ Der Berichterstatter erinnerte an die zahlreichen Fachkongresse, die sich bis zum Inkrafttreten des Bundesbeamtengesetzes bereits mit der ,beamtenrechtlichen Frage de lege lata und de lege ferenda eingehend befaßt haben“. $42 \mathrm{Da}$ man gleichwohl dieses Thema gewählt hat, wurde deshalb ausdrücklich damit erklärt, ,daß es durch einen bestimmten, geradezu aktuellen Vorfall ausgelöst worden ist: Die Entscheidungen des BVerfG zum Gesetz zu Art. 131 GG. “43

In diesen Urteilen wandte sich das BVerfG gegen die „Identitätstheorie“, freilich ohne dies dezidiert auszudrücken: Nach einer gründlichen Auseinandersetzung mit dem nationalsozialistischen Beamtenrecht und der dazu vertretenen Staatsrechtslehre stellte es lapidar fest:

„Alle Beamtenverhältnisse sind am 8. Mai 1945 erloschen.“44

Zu Art. 33 Abs. 5 GG führt das Gericht unter Verweis auf die zitierte These von Grewe aus:

„Er [Art. 33 Abs. 5 GG] gewährleistet vielmehr das Berufsbeamtentum als Einrichtung insoweit, als es sich in seiner hergebrachten Gestalt in den Rahmen unseres heutigen Staatslebens einfügen läßt." “45

Der Parlamentarische Rat, so das BVerfG, wollte dem Gesetzgeber bei der Gestaltung des Beamtenrechts Spielraum lassen, die hergebrachten Grundsätze des Berufsbe-

torisch Grotkopp, Beamtentum und Staatsformwechsel, Die Auswirkungen der Staatsformwechsel von 1918, 1933 und 1945 auf das Beamtenrecht und die personelle Zusammensetzung der deutschen Beamtenschaft, 1992.

41 Berichte von v. d. Heydte/Dürig, Perels und Naumann/Spanner, VVDStRL Berlin 1955. Zu Diskussion Kirn, Verfassungsumsturz oder Rechtskontinuität?, Die Stellung der Jurisprudenz nach 1945 zum Dritten Reich, insbesondere die Konflikte um die Kontinuität der Beamtenrechte und Art. 131 Grundgesetz, 1972.

42 Naumann (Fußn. 41), VVDStRL 1955, S. 89, spricht neben anderen Fachtagungen ausdrücklich die Referate des 39. DJT an.

43 Ders., ebda., S. 90; gemeint waren die Urteile des BVerfG vom 17.12.1953, BVerfGE 3, 58, 162, 187, 208.

44 BVerfGE 3, 58.

45 BVerfGE 3, 58, 137. Auf der 39. Tagung des DJT äußerte sich Selbert, Mitglied des Parlamentarischen Rats zur Frage der Berücksichtigung der hergebrachten Grundsätze: „Diese Formulierung bedeute viel weniger, als vielleicht viele von Ihnen dahinter suchen: Ganz bewußt hat der parlamentarische Rat damals den Ausdruck, wohlerworbene Rechte' vermieden. Ganz bewußt ist er ausgewichen in eine so farblose Formulierung ,Berücksichtigung hergebrachter Grundsätze.“ (Fußn. 31, S. D 106) 
amtentums sollten dabei ,berücksichtigt' nicht aber unter allen Umständen ,beachtet' werden." 46

Insgesamt scheinen die Staatsrechtslehrer Mitte der 1950er Jahre von der andernorts hochgelobten ${ }^{47}$ Auseinandersetzung des BVerfG mit Vergangenheit und Zukunftsgestaltung des Beamtentums noch nicht so recht überzeugt gewesen zu sein. Möglicherweise hat man auch die praktischen Auswirkungen des Urteils überschätzt. Schließlich war zu diesem Zeitpunkt ,die Inkorporation des alten Beamtenapparats schon fast vollständig abgeschlossen“.48 Auf der, wie er es nannte „kühlen Plattform der Staatsrechtslehrertagung" versuchte der Berichterstatter Naumann, die „historische Einmaligkeit“ des "deutschen Zusammenbruchs" von 1945 zu relativieren - schon mit dem für das Tagungsthema gewählten Plural „Staatsrechtskrisen“ wären bewußt Parallelen zwischen 1919 und 1945 gezogen worden: Weil das deutsche Beamtentum durch die „Bindung an die Staatsidee" charakterisiert wurde, war es zum Ende der Monarchie 1919 begreiflich,

„wenn man sich Sorge um den Bestand des Berufsbeamtentums deshalb machte, weil die Staatsidee nach 1919 an Geltung verloren zu haben schien. Deshalb sprach man mit Recht auch von einer Krise des Beamtentums, wie von einer Krise der Staatsidee, weil die Gemeinschaftsgrundlage zersetzt erschien.“49

In diesem Zusammenhang erinnerte der Berichterstatter an die Tagung der Deutschen Staatsrechtslehrer 1931, die dem Thema „Entwicklung und Reform des Beamtenrechts" galt. Deren Leitsätze ${ }^{50}$ werden als nach wie vor maßgebend beurteilt. Darin hieß es, daß

„die Problematik des Beamtenrechts grundsätzlich aus einer solchen des individuellen Beamtenverhältnisses zu einer Problematik der verfassungsrechtlichen Stellung des Beamtentums umgestaltet worden [ist]." 51

In der an die Berichte 52 anschließenden Aussprache hob damals Koellreutter die „Aufstellung der ,institutionellen Garantien'“ durch Schmitt hervor, die er für „sehr bedeutsam und wertvoll“" hielt. Er schloß als deren Konsequenz die Auslegung nicht aus,

46 Ders., a.a.O.

47 S. die Zitate bei Naumann (Fußn. 41), VVDStRL 1955, S. 94.

48 Perels (Fußn. 41), VVDStRL 1955, S. 367; von den 53.000 bis 1946 in den Westzonen von den Alliierten entlassenen Beamten waren bereits 52.000 wieder im Dienst, mit Nachweisen ders., ebda., S. 357.

49 Ders., ebda., S. 107; Grotkopp, Beamtentum und Staatsformwechsel (Fußn. 40), S. 187, 189, belegt historisch, daß seitens der Alliierten 1949 festgestellt werden konnte: „From 40 to 80 per cent of the officials in many branches of public administration are now reinstated former Nazis.“

50 VVDStRL 1931, Heft 7, Berlin und Leipzig 1932.

51 Gerber, Entwicklung und Reform des Beamtenrechts, ebda., S. 52, Sperrung im Original.

52 Ebenso wie bei der StRL-Tagung 1955 wurde auch 1931 ein österreichisches Koreferat (Merkl) gehalten, in dessen 1. Leitsatz es heißt (ebda., S. 102): „Die Erhaltung eines parteipolitisch neutral wirkenden Berufsbeamtentums als Brücke über die parteipolitische Zerrissenheit des deutschen Volkes ist rechtspolitische Aufgabe für die ganze, staatlich gespaltene deutsche Nation." Zur Bedeutung von Adolf Merkl für das österreichische Staatsrecht Wiederin, Denken vom Recht her, Über den modus austriacus in der Staatsrechtslehre, in: Schulze-Fieliz (Hrsg.), Staatsrechtslehre als Wissenschaft, Die Verwaltung, Beiheft; Bd. 7 2007, S. 293 ff., insbes. S. 301. 
daß die in Art. 129 Abs. 1 S. 3 der Weimarer Reichsverfassung enthaltenen „'wohlerworbenen Rechte' nur im Rahmen der institutionellen Garantie, nicht als Selbstzweck anerkannt und gesichert sind." 53

Auf der Suche nach Kontinuität wird 1954 aber nicht nur allgemein auf die Ergebnisse der Staatsrechtslehrertagung von 1931 rekurriert; die „institutionelle Garantie“ aus den 1930er Jahren wird ohne weiterer Reflektion übernommen. Für Naumann ließ selbst das „DBG von 1937 den gesamten traditionellen Gehalt des Staatsbeamtenverhältnisses noch deutlich erkennen“, die

,juristische Besonderheit der deutschen Staatskrise nach 1945 [wird] - vielleicht entscheidend- dadurch charakterisiert, daß sich an den Zusammenbruch eine vollständige Okkupation des Landes und Übernahme der Staatshoheit seitens der Siegermächte anschloß. "54

Dies weist schon auf den eigentlichen Kern der Mißbilligung der Entscheidung des BVerfG, die in der Praxis sogar dazu führte, daß sich Gerichte

„offen gegen das BVerfG gestellt [haben], weil sie die Beamten- und Soldatenverhältnisse 1945 nicht beendet sehen und den Betroffenen entsprechende Beschäftigungs- und Versorgungsansprüche sichern wollten." 55

Die Wissenschaft lieferte die Argumente: Weil staatsrechtlich der Fortbestand der Institution des Berufsbeamtentums 1945 davon abhängig war, ob die zur staatsrechtlichen Neuordnung befugte Autorität diese aufgehoben hat oder nicht, und weil die „Siegermächte erkennen ließen“, daß die Institution von ihnen ,nicht beseitigt werden sollte und nicht beseitigt worden ist", kann im Leitsatz 6 der Staatsrechtslehrertagung formuliert werden:

„Deshalb hat staatsrechtlich betrachtet, das deutsche Berufsbeamtentum die Staatskrise überdauert, mag auch historisch-politisch Diskontinuität angenommen werden." $" 56$

Forsthoff brachte die Kritik schließlich auf den Punkt: „Diskontinuität“ bedeutet für ihn staatsrechtlich die „Lossagung von den Verbindlichkeiten des vorangegangenen Regimes“, die durch eine ,hoheitliche, hochpolitische Entscheidung erst geschaffen wird." Weil aber die politischen Instanzen in Deutschland nach 1945 diese Entscheidung „bekanntlich nicht getroffen haben“, ist für ihn das Urteil des BVerfG

„deshalb ein den gegenwärtigen Justizstaat charakterisierender, apokrypher Souveränitätsakt."

53 Koellreutter, ebda., S. 117. 20 Jahre später griff Keßler in der Diskussion auf dem 39. DJT (Fußn. 31) diese Auffassung an (S. 102): „Der Begriff der institutionellen Garantie ist verbraucht (...) Er wurde damals gegen die Beamten vertreten, als es darum ging, die seit alters auf Lebenszeit angestellten Beamten in den Ruhestand zu versetzen und man nach einem Grund suchte, wie es zu machen sei.“ Rechtshistorisch dazu Spranger, Die Stellung des Beamten in der Weimarer Republik, Ein Beitrag zum verfassungsrechtlichen Vorverständnis des Art. 33 Abs. 5 GG, 2007.

54 Naumann (Fußn. 41), VVDStRL 1955, S. 93

55 Dies wird von Schlink, Abschied von der Dogmatik, Verfassungsrechtsprechung und Verfassungsrechtswissenschaft im Wandel, JZ 2007, S. 157 ff., als Ausnahmebeispiel dafür genannt, daß Gerichte sich gegen das BVerfG gestellt haben. (S. 158)

56 Ders., ebda., S. 118. 
Auch wer der verführerischen Wortschöpfung des „,apokryphen Souveränitätsakts“ nicht folgen will, wird doch zugeben müssen, daß es nicht zuletzt die Rechtsprechung des BVerfG ${ }^{58}$ gewesen ist, die dem Berufsbeamtentum immerhin die Kontinuität der Versorgung auf „amtsangemessenen“ Niveau über die verschiedensten Krisen hinweg gesichert hat.

\section{Beamtentum, aktuelle Reformversuche und das Bundesverfassungsge- richt}

Als man sich beim Vereinigungsprozeß beider deutscher Staaten 1990 darauf verständigte, die überkommene Beamtenversorgung der Bundesrepublik von der Übernahme neuer Verpflichtungen freizustellen, ${ }^{59}$ geschah dies weitgehend auf Kosten der gesetzlichen Rentenversicherung. ${ }^{60}$ Es war allerdings bald abzusehen, daß die dadurch bei der Rentenversicherung erforderlichen Einsparungen die Beamtenversorgung politisch nicht gänzlich unberührt lassen konnten.

\section{Beamtenrecht versus Beamtentum}

Seit 1990 hat der Gesetzgeber dann auch einige „kostensenkende Reformmaßnahmen aus der gesetzlichen Rentenversicherung wirkungsgleich auf die Beamtenversorgung übertragen“.61 Allerdings stieß er dabei trotz des 53. Gesetzes zur Änderung des

57 Forsthoff, in der Aussprache, ebda., S. 161.

58 Freilich nicht nur das BVerfG, ,auch das Bundesverwaltungsgericht hat in einer überaus feinsinnigen Entscheidungspraxis über die Jahrzehnte hinweg jeglichem Anpassungsdruck widerstanden.“ Kühling, Hergebrachte Grundsätze, myops 6/2009, S. 4 , 7. Zur „ungewöhnlichen Vielzahl von Entscheidungen des öffentlichen Dienstrechts“ Kenntner, Aktuelle Rechtsprechung des Bundesverfassungsgerichts zum Beamtenrecht, JZ 2008, S. 340 ff. Schließlich ist aber auch der BGH zu nennen, der z.B. betr. des Widerrufs der Zulassung zum Rechtsanwalt wegen der Ernennung zum Fachhochschullehrer die beamtenrechtliche Argumentation von BVerfG und BVerwG übernahm. Die Ernennung zum Beamten auf Lebenszeit bedeutet auch für den BGH ,ein öffentlich-rechtliches Dienstund Treueverhältnis zum Staat“, das der Idee des freien Berufs Rechtsanwalt mit dem dafür „unbedingt erforderlichen rechtlichen und tatsächlichen Handlungsspielraum“ entgegensteht, s. zuletzt BGH, Beschluß vom 6.7.2009, AnwZ (B) 52/08.

59 Zum Prozedere und dessen problematischen Folgen Köhler, Völker- verfassungs- und sozialrechtliche Probleme bei der Überführung von DDR-Zusatz- und Sondersystemen in die gesetzliche Rentenversicherung, Neue Justiz 1993, S. 4 ff.

60 S. statt vieler Ritter, Der Preis der deutschen Einheit, Die Wiedervereinigung und die Krise des Sozialstaats, 2. erw. Aufl. 2007; Fuchs/Köhler, Verfassungswidrigkeit der vorgezogenen Anhebung der Altersgrenzen bei der Rente wegen Arbeitslosigkeit, SGb 2002, S. 645 ff.

61 Färber/Funke/Walther (Fußn. 2), DÖV 2009, S. 134; Battis, Entwicklung des Beamtenrechts seit 2008, NVwZ 2009, S. 812 ff., und Körtek, Die Beamtenversorgung in der Bundesrepublik Deutsch- 
Grundgesetzes vom 28. August 2006 bald an die Grenze der „hergebrachten Grundsätze des Berufsbeamtentums." Mit diesem Gesetz erweiterte der Gesetzgeber zwar den Art. 33 Abs. 5 GG dahin, daß ,,vor dem abschließenden Punkt die Wörter , und fortzuentwickeln' eingefügt" 62 wurden. Diese Neuerung hat aber dem Bundesverfassungsgericht zufolge am „maßgeblichen Regelungsgehalt der Vorschrift (...) nichts geändert“das Gericht folgert das daraus, daß

„nach der eindeutigen Gesetzesfassung allein das Recht des öffentlichen Dienstes, nicht aber der hierfür geltende Maßstab, die hergebrachten Grundsätze des Berufsbeamtentums"

\section{fortentwickelt werden können. ${ }^{63}$ Mit diesen „hergebrachten Grundsätzen“ wird}

„die Einrichtung des Berufsbeamtentums, wie es sich in der deutschen Geschichte herausgebildet und um seiner Funktion willen Eingang in die Verfassungsordnung der Bundesrepublik Deutschland gefunden hat, institutionell garantiert." "64

Am Grundgedanken der Lehre Schmitts von der „,institutionellen Garantie“, nämlich zwischen konkreten individuellen Freiheitsrechten und der Garantie von abstrakten „Einrichtungen“ zu unterscheiden, ${ }^{65}$ wird also trotz deren Problematik festgehalten. Schon zu Zeiten der WRV ist es ja nicht gelungen, die Grenze zwischen ,zulässiger Ausgestaltung und unzulässiger Aushöhlung“ durch Bezugnahmen auf selbst ausfüllungsbedürftige Begriffe wie das „Wesentliche“ oder das „historisch Überkommene“66 plausibel zu ziehen.

Das BVerfG hat in dieser Entscheidung, in der es die ,,antragslose Teilzeitbeschäftigung von Beamten ohne die Möglichkeit zur Wahl der vollen Beschäftigung“67 für verfassungswidrig erklärt, unter Bezug auf die eigene Rechtsprechung zwar anerkannt, daß

„nicht jede Regelung des Beamtenrechts, die sich als hergebracht erweist, (...) von der institutionellen Garantie erfaßt [wird]“. 68

land, in diesem Band, S. 47 ff.; speziell zur Anhebung der Altersgrenzen der Beamten Ruland, Zur Verfassungsmäßigkeit der Anhebung der Altersgrenzen im Rentenversicherungs- und Beamtenversorgungsrecht, in: Manssen u.a. (Hrsg.), Nach geltendem Verfassungsrecht, Festschrift für Udo Steiner zum 70. Geburtstag, 2009, S. 662 ff.

62 BGBL I 2006 S. 2034.

$\mathrm{Zu}$ gesetzgeberischem Prozedere und Inhalt der Änderung Höfling/Burkiczak, Die Garantie der hergebrachten Grundsätze des Berufsbeamtentums unter Fortentwicklungsvorbehalt, Erste Überlegungen zur Änderung von Art. 33 Abs. 5 GG, DÖV 2007, S. 328 ff.

63 BVerfGE 119, 247, 272.

64 BVerfGE 119, 247, 260.

65 Schmitt, Verfassungslehre, 1. Aufl. 1928, 4. Aufl. unveränderter Nachdruck 1965, S. 170: „Institutionelle Garantien sind von Grundrechten zu unterscheiden." Zu dieser Lehre, ihren Vorläufern und den aktuellen Meinungsstand umfassend Mager, Einrichtungsgarantien, Entstehung, Wurzeln, Wandlungen und grundgesetzmäßige Neubestimmung einer dogmatischen Figur des Verfassungsrechts, 2003, und Mehring, Carl Schmitt, Aufstieg und Fall, 2009, II. Teil, Weimarer Leben und Werk, S. 260 ff.: Vom klassischen Freiheitsrecht zur institutionellen Garantie.

66 Mager, ebda., S. 33.

67 Dazu ausführlich z.B. Kenntner (Fußn. 36), DVB1 2007, S. 1321-1328.

68 BVerfGE 119, 247, 262. 
Garantiert wird nicht das ,gewachsene Berufsbeamtenrecht, sondern das Berufsbeamtentum“. ${ }^{9}$ Beim Versuch, rechtlich zu präzisieren, was danach definitiv von der „Garantie“ erfaßt wird, bleibt unerwähnt, daß der Terminus „Berufsbeamtentum“ von den Nationalsozialisten als Gegensatz zu den angeblichen „Parteibuchbeamten“ des „Weimarer Systems“ geprägt und erstmals in einen Gesetzestext aufgenommen worden ist. ${ }^{70}$ Das BVerfG bezieht sich statt dessen ausdrücklich auf den oben erwähnten Beitrag von Thieme in der Studienkommission für die Reform des öffentlichen Dienstrechts 71 - freilich ohne dessen Kritik zu erwähnen oder gar anzunehmen. Denn das „Wesen einer Einrichtungsgarantie“ wird deren „Sinn“ entnommen, der „gerade darin liegt",

„den Kernbestand der Strukturprinzipien - mithin die Grundsätze, die nicht hinweggedacht werden können, ohne dass damit zugleich die Einrichtung selbst ihren Charakter grundlegend verändert würde - dem Gesetzgeber verbindlich als Rahmen vorzugeben.“72

Schwer faßbare Begriffe wie „Wesen“ und „Sinn“ einer Einrichtung werden, wie vor 40 Jahren, gerügt, wieder mit einem selbst nicht analysierten (und offenbar einer Analyse nicht zugänglichen) „Kernbestand der Strukturprinzipien“ erklärt, der dann im Zirkelschluß den ,grundlegenden Charakter der Einrichtung“ fixieren sollen. Die Entscheidung erging mit sechs zu zwei Stimmen.

In der Begründung seiner abweichenden Meinung findet Gerhardt dafür die Umschreibung, daß die von der Senatsmehrheit herangezogenen Grundsätze von dieser ,in einem bestimmten, gesetzgeberischer Konkretisierung nicht zugänglichen Sinn“ verstanden werden. ${ }^{73}$ Für die Praxis ${ }^{74}$ gilt damit, daß im Zweifel jeweils das BVerfG entscheidet, ob eine beamtenrechtliche Regelung die verfassungsrechtlich garantierte Institution Berufsbeamtentum verletzt.

\section{2. „,beachten“versus ,, berücksichtigen“-,,Modifizierung“versus ,,Veränderung“}

Auch in seinem Beschluß vom 20. März $2007^{75}$ griff das BVerfG auf die „hergebrachten Strukturprinzipien“ des Berufsbeamtentums zurück. Es stellte die „Versorgung aus dem letzten Amt“ als eine „Ausprägung“ des Alimentationsprinzips zusammen mit

69 BVerfG, a.a.O.

70 Mit Nachweisen der Fundstellen in der Literatur und die Textstelle im Gesetz zur Wiederherstellung des Berufsbeamtentums vom 7. April 1933 (RGB1. I, 41) Krause, Die hergebrachten Grundsätze des Berufsbeamtentums, Eine rechtshistorische Analyse, 2008, S. 6-8.

71 S. o. unter I 1 b.

72 BVerfGE 119, 247, 263.

73 BVerfGE 119, 247, 283.

$74 \mathrm{Zu}$ dieser aktuell Szalai, Beamte in Teilzeit und Versetzung von „Vollzeitbeamten“ - Ein Problem nicht nur in Brandenburg, DÖV 2009, S. $311 \mathrm{ff}$.

75 BVerfGE 117, 372. 
diesem in die Reihe dieser „Strukturprinzipien“ und schrieb damit eine Wartefrist nach $\S 5$ Abs. 3 S. 1 BeamtVG ${ }^{76}$ von höchstens zwei Jahren verfassungsrechtlich fest.

Das Gericht geht in seiner Argumentation zunächst einmal davon aus, daß sich im überkommenen „Grundsatz der Versorgung aus dem letzten Amt“ das „Alimentationsund das Leistungsprinzip überschneiden“, weshalb dieser Grundsatz ,zu den Grundlagen [gehört], auf denen die Einrichtung des Berufsbeamtentums beruht. “77 Er ist „,mithin“ vom Gesetzgeber „nicht nur zu berücksichtigen, sondern zu beachten“. 78 Diese feine Unterscheidung ist in der Rechtsprechung des BVerfG seit langem geläufig, ${ }^{79}$ obwohl semantisch ein Unterschied beider Aussagen vielleicht als Nuance zu spüren, nicht aber dingfest zu machen ist: So erläutert der Duden „beachten“ beispielhaft mit „etw. berücksichtigen, befolgen“ und „berücksichtigen“ umgekehrt mit „bei (...) seinem Handeln beachten" 80 - die Verben sind also synonym.

Noch schwieriger zu verstehen wird die Rechtsprechung des BVerfG dadurch, daß das Gericht aus diesen bedeutungsgleichen Wörtern nicht nur unterschiedliche Pflichten folgert, sondern diese wiederum mit Synonymen beschreibt. Ändert nämlich der Gesetzgeber den Grundsatz der Versorgung aus dem letzten Amt durch Einführung einer „Mindestverweildauer im Beförderungsamt“ erst auf ein Jahr, später auf zwei Jahre, so muß dies als Beachtung der Grundlagen des Berufsbeamtentums hingenommen werden, da es sich nicht um einen Eingriff in den Alimentationsgrundsatzes handelt, sondern nur um dessen „Modifizierung“:

„Eine Mindestverweildauer im Beförderungsamt ist somit - ebenfalls hergebrachter, lediglich modifizierender- Bestandteil des Bemessungsprinzips der Versorgung aus dem letzten Amt."“81

Demgegenüber handelt es sich bei einer Verlängerung der Wartefrist über diese Zeitspanne hinaus, im zu entscheidenden Fall war das eine Verlängerung von zwei Jahren auf drei Jahre,

„um eine grundlegende Veränderung, die sich nicht mehr als bloße Modifizierung der bisher anerkannten Einschränkungen des hergebrachten Grundsatzes der Versorgung aus dem letzten Amt erklären lässt 82

$76 \S 5$ Abs. 3 S. 1 BeamVG: „Ist ein Beamter aus einem Amt in den Ruhestand getreten, das nicht der Eingangsbesoldungsgruppe seiner Laufbahn oder das keiner Laufbahn angehört, und hat er die Dienstbezüge dieses oder eines mindestens gleichwertigen Amtes vor dem Eintritt in den Ruhestand nicht mindestens zwei Jahre erhalten, so sind ruhegehaltfähig nur die Bezüge des vorher bekleideten Amtes." Der Gesetzgeber wollte die Frist auf drei Jahre verlängern.

77 BVerfGE 117, 372, 382

78 BVerfGE 117, 372, 383.

79 Die ausführlichen Rechtsprechungszitate der ,stRspr.“ durch das BVerfG selbst, a.a.O.

80 Duden, Das große Wörterbuch der deutschen Sprache in zehn Bänden, 3. Aufl. Bd. 1, lemmata „beachten“, „berücksichtigen“. Von daher ist nicht ganz nachvollziehbar, warum in den Kommentaren zum GG die Unterscheidung des BVerfG weitgehend akzeptiert wird, s. etwa Lübbe-Wolff, in: Dreier (Hrsg.), Grundgesetz-Kommentar, Bd. 2, 1998, Art 33 Rdnr. 74: „'Berücksichtigen’ bedeutet, für sich genommen, nicht dasselbe wie ,beachten'“.

81 BVerfGE 117, 372, 383. 
Läßt man die (eigentlich verdeckt signifikanten ${ }^{83}$ ) Adjektive „grundlegend“ (= Veränderung) und „,bloß“ (= Modifizierung) beiseite und bemüht sich um Aufklärung der genauen sprachlichen Bedeutung, so wird das Wort Änderung mit „Umgestaltung“ und „Modifikation“ erklärt, ${ }^{84}$ das Wort Modifikation wiederum mit „Abweichung, Abänderung" ${ }^{\prime 85}$ - ein weiteres Mal soll also gesetzgeberisches Handeln diesseits oder jenseits des verfassungsrechtlich Zulässigen durch Synonyme differenziert werden!

\section{3. „,Traditionsbildender Zeitraum“versus Regelungsbedürfnisse der Gegenwart}

Die Karenzzeit von einem Jahr muß nach dem BVerfG auch deshalb zu den „hergebrachten“ Grundsätzen des Berufsbeamtentums gezählt werden, weil sie im „traditionsbildenden Zeitraum entwickelt" worden ist. ${ }^{86}$ Damit ist, wie oft übersehen wird, ${ }^{87}$ die zweifache vom BVerfG verlangte Voraussetzung für eine Festschreibung „,hergebrachter Grundsätze“ gemeint, daß diese sich nämlich durch „Fundamentalität“ und durch „Traditionalität“ auszeichnen müssen. Nun ist „Traditionalität“ kein unzweideutiger, aus sich selbst heraus verständlicher Begriff - der Duden verzeichnet ihn überhaupt nicht ${ }^{88}$ _ obwohl er in der Rechtsprechung des BVerfG bereits einige Tradition hat.

Anders als das BVerfG versucht Glenn in seinem preisgekrönten Werk „Legal Traditions of the World" 89 eingehend, eine Antwort auf die Frage zu geben, was (speziell im Recht) Tradition zur „Tradition mit Rechtswirkung“ macht. Ein eindeutiges, überall

82 BVerfGE 117, 372, 385.

83 Entspräche eine „bloße“ Veränderung einer Modifizierung und wäre dann hinzunehmen? Gibt es Veränderungen, die nicht ,grundlegend“ sind?

84 Duden (Fußn. 80), lemma „Änderung““

85 Ebda., Bd. 6, lemma „Modifikation“. Ein Beispiel dafür, wie sehr man sich daran gewöhnt hat, daß verfassungsrechtliche Grenzen vom „Sprachgefühl“ gezogen werden, findet sich aktuell Ruland, in: Manssen u.a. (Hrsg.), Nach geltendem Verfassungsrecht (Fußn. 61), S. 677: „Ihm [i. e. der Gesetzgeber] standen schon vor dieser Änderung Freiräume zu, die sich zum einen aus der Natur des Regelungsauftrags, zum anderen aus seiner nur auf die ,Berücksichtigung' begrenzten Bindung an die hergebrachten Grundsätze ergeben.“

86 BVerfGE 117, 372, 375.

87 Zum Vorwurf, daß die Rechtsprechung ,das kumulative Verhältnis dieser beiden Anforderungen immer wieder aus den Augen verloren hat", s. Lübbe-Wolff, in: Dreier (Hrsg.), GrundgesetzKommentar (Fußn. 80), Art 33 Rdnr. 70.

88 Duden (Fußn. 80), Bd. 9, verzeichnet zwar das lemma „Tradition“ und 17 damit verbundene Begriffe, darunter „Traditionalismus“, dies ist aber „eine geistige Haltung, die an der Tradition festhält" und das ist mit „Traditionalität“ offenbar nicht gemeint. Weil bei der Frage nach „Rechtlicher Tradition" die Entstehung von Recht interessiert, tritt Fikentscher in seinem einleitenden Beitrag zu $\mathrm{Fi}$ kentscher u.a. (Hrsg.), Entstehung und Wandel rechtlicher Traditionen, 1980, S. 27, dem Mißverständnis entgegen, daß dabei „einem juristischen Traditionalismus das Wort geredet" wird.

89 Glenn, Legal Traditions of the World, Sustainable Diversity in Law, 2. Aufl. 2004; der Erstauflage wurde 1998 der Grand Prize der International Academy of Comparative Law verliehen. Eine ungewöhnlich gründliche Rezension des Werks geben Foster u.a., A Fresh Start for Comparative Legal Studies?, JCL 1. 1, S. 100 ff. 
gültiges Merkmal dafür scheint es nicht zu geben. Zum Problem der Möglichkeit einer "Theorie der Tradition"90 zitiert Glenn deshalb die sehr zurückhaltende Aussage Poppers, der im ersten Satz seines Essays „Toward a Rational Theory of Tradition“ meinte, „the emphasis should be on the word ,towards" “.91

Das BVerfG geht auf die theoretische Problematik nicht ein. Es erklärt nicht, warum durch Zeitablauf Vorrechte zu Recht werden sollen, es verlangt ohne weitere Begründung für das Entstehen von „Tradition“ nur, daß die Grundsätze

„mindestens unter der Reichsverfassung von Weimar als verbindlich anerkannt und gewahrt worden sind."92

Offen bleibt also auch, ob, wann und wodurch eine Tradition einmal ein Ende finden kann; im Grunde folgt aus dieser vagen Festlegung eigentlich nur, daß „erst unter der Geltung des Grundgesetzes herausgebildete Grundsätze nicht zum institutionell gewährleisteten Bestand gehören."93

In der hier diskutierten Entscheidung steckt das Gericht diesen das geltende Recht qualifizierenden Zeitraum durch eine aleatorisch wirkende Darstellung des Werdens und Vergehens des „Versorgungsansatzes am letzten Amt“ zeitlich jedoch sehr viel weiter zurück in die Vergangenheit ab - beginnend mit dem „Gesetz betreffend die Rechtsverhältnisse der Reichsbeamten vom 31. März 1873“, über diverse noch ältere „Civilstaatsdienergesetze“ einzelner Staaten (Preußen, Sachsen, Württemberg94) bis hin zum Deutschen Beamtengesetz von 1937 (DBG), das erstmals für das „allgemeine Beamtenrecht" bestimmte,

„daß sich Pensionen eines Beamten, der die Bezüge aus einem Beförderungsamt nicht mindestens ein Jahr erhalten hatte, aus dem Diensteinkommen des zuvor bekleideten Amtes berechnete." "95

Das Gericht bemerkt zu dieser Vorschrift nur knapp, daß sie „der Verhinderung von Gefälligkeitsbeförderungen“ diente, ${ }^{96}$ um sich dann sofort dem Bundesbeamtengesetz

90 Ders., ebda., S. 3: „Is a theory of tradition then possible?“

91 Popper, Toward a Rational Theory of Tradition, in: Popper, Conjectures and Refutations. 3. Aufl. 1969 , S. $120 \mathrm{ff}$.

92 BVerfG 8, 332, 343. Das BVerfG nimmt für seine Rechtsprechung zum Beamtentum nicht zur Kenntnis, was seit der Aufklärung gilt: „Je offenkundiger die souveräne Gesetzgebungsinstanz [...] des Staates einen Monopolanspruch erhebt und auch einlöst, um so mehr müssen Gewohnheitsrecht, Herkommen und Brauch an rechtlicher Bedeutung verlieren." Wiedenhöfer, Lemma Tradition, Traditionalismus, in: Brunner u.a. (Hrsg.), Geschichtliche Grundbegriffe, Historisches Lexikon zur politisch-sozialen Sprache in Deutschland, Bd. 6 1990, S. 607, 624.

93 Lübbe-Wolff, in: Dreier (Hrsg.), Grundgesetz-Kommentar (Fußn. 80), Art 33 Rdnr. 71, Fußn. 363.

94 BVerfG 8, 373-375. Die bayerische „Hauptlandespragmatik über die Dienstverhältnisse der Staatsdiener vorzüglich in Beziehung auf ihren Stand und Gehalt" vom 1.1.1805 wird nicht erwähnt, obwohl damit ,zum ersten Mal Konzeptionen eines modernen Beamtentums Gesetz geworden [sind], die zwei zentrale Postulate umschlossen: die Unkündbarkeit der Beamten und die Garantie einer Versorgung für sie“, Zacher, Die Versorgung der Beamten, Richter und Soldaten, 1984, S. 7.

95 BVerfGE 117, 375.

96 Ebda. mit Hinweis auf Fischbach, BBG, 2. Aufl. 1956, S. 809 ff. 
(BBG) von 1953 zuzuwenden, das genau diese (und nicht nur diese, soweit sie nicht nationalsozialistisches Gedankengut enthielten) Regelung des DBG übernahm. Angesichts der entschiedenen Distanzierung des BVerfG von der Ausgestaltung des Beamtentums in der NS-Zeit ${ }^{97}$ verwundert das Abstellen auf den rechtstechnischen, offenbar als wertneutral beurteilten Inhalt eines Gesetzes, das in $\S 1$ Abs. 2 den Beamten zum

„Vollstrecker des Willens des von der Nationalsozialistischen Deutschen Arbeiterpartei getragenen Staates"

erklärt hat. ${ }^{98}$ Die Entscheidung macht so vor allem deutlich, wie schwer es angesichts (nicht allein) der jüngeren ${ }^{99}$ deutschen Geschichte ist, für das verfassungsrechtlich relevante Verfestigen einer Norm einen ,"ängeren, Tradition bildenden Zeitraum“ zu erkennen. Dies gilt um so mehr, als die demokratische Zeitspanne der deutschen Vergangenheit kurz ist, die der Gegenwart unter dem Grundgesetz dagegen immer länger wird und bereits selbst Traditionen bildet, ${ }^{100}$ die von der Rechtsprechung auf Dauer wohl nicht ignoriert werden können.

Die Richter Osterloh und Gerhardt weisen so auch in ihrer vom Beschluß des Zweiten Senats abweichenden Meinung darauf hin, daß sich die

97 S. z.B. die oben erwähnte Entscheidung BVerfGE 3, 58, und später BVerfGE 6, 132.

98 RGB1 I S. 39; in der Präambel des DBG hieß es: „Ein im deutschen Volk wurzelndes, von nationalsozialistischer Weltanschauung durchdrungenes Berufsbeamtentum, das dem Führer des Deutschen Reichs und Volkes, Adolf Hitler, in Treue verbunden ist, bildet einen Grundpfeiler des nationalsozialistischen Staates."

Die Vorgabe eines „von nationalsozialistischer Weltanschauung durchdrungenes Berufsbeamtentums" wurde dem Gesetz zur Wiederherstellung des Berufsbeamtentums entnommen (s. o. I 3 a). Die Ideologie des Nationalsozialismus betreffende Bestimmungen wurden durch Verordnungen der Zonenbefehlshaber unmittelbar nach der Übernahme der Regierung durch die Alliierten aufgehoben. Das BVerfG scheint das zu spüren, denn es spricht später (BVerfGE 117, 383) ungewohnt ungenau vom ,,in den vor dem Jahr 1945 erlassenen Vorschriften enthaltenen ,Einjahresschritt' $(\ldots)^{\text {“. }}$.

99 Exemplarisch dafür Leben und Werk von Arthur Brand, einem der wichtigsten Kommentatoren des Beamtenrechts im Kaiserreich, in der Weimarer Republik und der NS-Zeit, dessen „Texte jedenfalls der Weimarer Zeit (...) bestes Material zur Feststellung hergebrachter Grundsätze des Berufsbeamtentums [bieten]“, dessen „engagierte Wiedergabe nationalsozialistisch strukturierter Elemente der ersten gemeindeutschen Beamtenrechtskodifikation indes ex negativo die Nachgeborenen zu kritischer Reflexion [mahnen]. "So Günther, Arthur Brand und das Beamtenrecht, Ausgewählte Aspekte des Dienstrechts im Kaiserreich, während der Weimarer Republik, der NS-Diktatur, Der Staat 2009, S. $411,457$.

100 Glenn, Legal Traditions of the World (Fußn. 89), S. 6 ff., diskutiert dies unter „Instant Tradition“; weil es ,the most obvious and generally accepted element of tradition“ ist, zitiert er T. S. Eliot, der als das wesentliche jeder „Tradition“ deren „pastness“ nannte (Eliot, Tradition and the Individual Talent, in: The Sacred Wood: Essays on Poetry and Criticism, London 1922). Ein Hinweis auf die für einen Rechtsvergleich darin auch liegende sprachliche Schwierigkeit folgt aus dem ersten Satz dieses berühmten Essays: „In English writing we seldom speak of tradition, though we occasionally apply its name in deploring its absence.“ Neben ,pastness“ verlangt Glenn „,normative authority, and active involvement of the recipients" als zentrale Elemente (S. $40 \mathrm{ff}$.) 
„rechtlichen und tatsächlichen Verhältnisse seit der Entscheidung des Senats vom 7. Juli 1982 zur Wartefrist von zwei Jahren wesentlich geändert haben“101

Sie erinnern daran, daß zwischen 1963 und 1993 eine nicht unerhebliche Verringerung des Abstands zwischen dem Durchschnittsverdienst während einer Beamtenlaufbahn und dem Verdienst im Spitzenamt zu verzeichnen, eine Verlagerung der Laufbahngruppen vom einfachen und mittleren Dienst zum gehobenen und höheren Dienst festzustellen sowie die mit dem Dienstrechtsreformgesetz von 1997 eingeführte Verlangsamung des Besoldungsanstiegs durch anfängliche Beschleunigung und Erhöhung der Besoldungszuwächse kompensiert worden ist. Hinsichtlich der Wartefrist bedeutet dies, daß sich

„der Beamte typischerweise bereits in dem davor liegenden Zeitraum seinem bisherigen Status entsprechend am gesellschaftlichen Leben beteiligt und eingerichtet hat."102

Nur wenig überspitzt ließe sich sagen, daß, je mehr ein beamtenrechtlicher Grundsatz „hergebracht“ ist im Sinne des vom BVerfG vage genug bestimmten ,traditionsbildenden Zeitraums“, desto weniger scheint er den Regelungsbedürfnissen der Gegenwart zu entsprechen.

101 BVerfGE 117, 372, 403. Zur Besonderheit der "Sondervoten" zuletzt Voßkuhle, Stabilität, Zukunftsoffenheit und Vielfaltssicherung, JZ 2009, S. 917, 922: „Zwar kündigt sich in Sondervoten nicht immer die neue Verfassungshorizonte aufreißende und die Verfassungsinterpretation voranbringende ,Meinung von morgen' an, man sollte die Bedeutung bestimmter Sondervoten für eine spätere Fortschreibung oder gar Änderung der Rechtssprechung aber nicht zu gering schätzen.“

102 BVerfGE 117, 372, 404. 